\title{
An Optimization to Schedule Train Operations with Phase-Regular Framework for Intercity Rail Lines
}

\author{
Huimin Niu and Minghui Zhang \\ School of Traffic and Transportation, Lanzhou Jiaotong University, Lanzhou 730070, China \\ Correspondence should be addressed to Huimin Niu, hmniu@mail.lzjtu.cn
}

Received 7 September 2012; Accepted 15 October 2012

Academic Editor: Wuhong Wang

Copyright (c) $2012 \mathrm{H}$. Niu and M. Zhang. This is an open access article distributed under the Creative Commons Attribution License, which permits unrestricted use, distribution, and reproduction in any medium, provided the original work is properly cited.

The most important operating problem for intercity rail lines, which are characterized with the train operations at rapid speed and high frequency, is to design a service-oriented schedule with the minimum cost. This paper proposes a phase-regular scheduling method which divides a day equally into several time blocks and applies a regular train-departing interval and the same train length for each period under the period-dependent demand conditions. A nonlinear mixed zero-one programming model, which could accurately calculate the passenger waiting time and the in-train crowded cost, is developed in this study. A hybrid genetic algorithm associated with the layered crossover and mutation operation is carefully designed to solve the proposed model. Finally, the effectiveness of the proposed model and algorithm is illustrated through the application to Hefei-Wuhan intercity rail line in China.

\section{Introduction}

Intercity rail lines, as a rapid transport mode connecting two cities, have been paid much attention by the governments all over the world. They have become one of the most important engines for boosting regional economic development and accelerating the urbanization process. In recent years, great importance has been attached to their construction in China. The associated rail networks connecting many important cities have been built or are under construction in several economically developed regions, such as Pearl River zone and Yangtze River zone, and there is a current trend for them to expand to the rest of the country.

An intercity rail line, either in matters of passenger demands or operation scheduling, is different from the general one. For intercity rail lines, the operations are characterized with rapid speed and high frequency, and the process of passengers arriving at the stations are time-dependent and stochastic. An even schedule with a constant headway between consecutive trains may result in long passenger waiting times during oversaturated periods, 


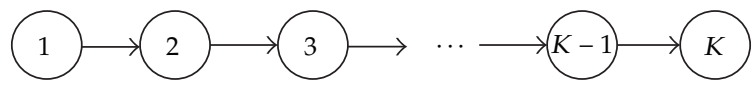

Figure 1: The illustration of an intercity rail line.

or ineffective train capacity utilization during unsaturated periods due to the undercapacity. A train schedule with inconstant headways, however, may lead to the frequent adjustment of predetermined schedule and the complexity of operations management. There is also trouble in determining the number of cars constituting a train, which should not be always steady or frequently modified.

Various attempts have been made to obtain a train schedule for railroad transports using optimization methods in the past decades. Ghoneim and Wirasinghe [1] defined the total cost as the sum of the travel time and the relevant rail capital and operating cost and designed a train scheduling plan with the minimum cost. Goossens et al. [2] introduced several models for solving operational scheduling problems in which railway lines can have different halting patterns. Liebchen [3] adapted a periodic event-scheduling approach and a well-established graph model to optimize the Berlin subway timetable. Claessens et al. [4] developed a mathematical programming model subjected to service and capacity constraints to optimize train operations. Ghoseiri et al. [5] built a multiobjective optimization model for the passenger train scheduling problem on a rail network which includes single and multiple tracks, as well as multiple platforms with different train capacities. Khan and Zhou [6] developed a stochastic optimization formulation for incorporating segment travel-time uncertainty and dispatching policies into a medium-term train-timetabling process, which is aimed to minimize the total trip time in a published timetable and reduce the expected schedule delay. Zhou and Zhong [7] formulated train scheduling models which consider segment and station headway capacities as limited resources, and developed algorithms to minimize both the expected passenger waiting times and total train travel times. Nachtigall and Voget [8] discussed the cost benefit between the investigation for reforming track states and the quality of the resulting timetable measured by the remaining waiting times. Palma and Lindsey [9] analyzed the schedule delay costs incurred from travelling earlier or later than desired and formulated an optimization model with the objective of minimizing the total riders' schedule delay costs. Nguyen et al. [10] presented a graph theoretic framework for the passenger assignment problem. Wong et al. [11] presented a mixed-integer-programming model for the schedule synchronization to minimize passengers' transfer times. Meng and Zhou [12] established a robust single-track train dispatching model under a dynamic and stochastic environment. Carey and Crawford [13] formulated a train scheduling model on a network of busy complex stations and designed a series of heuristics for finding and resolving train conflicts so as to satisfy various operational constraints and objectives. Caimi et al. [14] constructed a resource-constrained multicommodity flow model for conflict-free train routing and scheduling. Chang et al. [15] built a multiobjective programming model for the optimal allocation of passenger train services on an intercity high-speed rail line without branches.

The above-mentioned studies provide useful methods for the optimization of train schedule for railway networks during a particular time period. Nonetheless, research to date has focused primarily on scheduling problem with even headways, and a unified framework for scheduling methods that can consider uneven headways and time-dependent demand patterns is critically limited. This paper focuses on an intercity rail line and proposes a phase-regular scheduling method, which divides a day equally into several time blocks and 
applies the regular train-departing interval and the same train length during each period. An optimization model is presented to analytically calculate the passenger waiting time and the in-train crowded cost under a dynamic demand condition.

The remainder of this paper is organized as follows. An optimization model to the train scheduling problem for an intercity rail line is given in Section 2. In Section 3, a genetic algorithm procedure with two-layer framework is presented. In Section 4 , there is a numerical example provided to illustrate the application of the proposed model and algorithm. The last section brings the paper to a conclusion and outlines the possibilities for future research in related areas.

\section{Formulation}

\subsection{Problem Statement}

This study considers the train operations along one direction at an intercity rail line which consists of $K$ stations as shown in Figure 1. The stations along the direction are numbered as $1,2, \ldots, K$, and $i$ is used to index a station. Assume that all trains have the same speed between two consecutive stations and $R^{i}$ is used to denote the running time between station $i$ and $i+1$.

The passengers traveling by intercity rail lines arrive at the stations randomly, and they wait for the latest arriving train and then reach their destinations. The demands associated with intercity rail lines are characterized as being dynamic and stochastic. In terms of demand, the travel purposes of passengers are mainly for work and business, and the phase aggregation is significant. Based on such considerations, this paper divides a day equally into several time blocks (e.g., 1 hour as a period) and uses $\tau$ to denote a period and $\mathfrak{R}$ to represent the set of periods $(\tau \in \mathfrak{R})$. At the same time, this study uses $D^{i, i+s}(\tau)$ to denote the number of passengers who arrive at station $i$ during period $\tau$ travelling to station $i+s$.

Based on the characteristics of passenger demands and train operations about the intercity rail systems, a phase-regular train schedule, which has an even headway and the same train length during each period, is adopted in this study. According to the operation practice of intercity rail systems, this paper also assumes that there are only two train patterns, namely, the large pattern and the small pattern, in order to simplify the problem. The large pattern has more cars (e.g., 6 cars) and the small pattern has fewer cars (e.g., 4 cars) to form a train. To design a train schedule is actually to determine the number of departed trains and the associated train pattern for each period. The decision variables are defined as follows:

$x(\tau)$ : number of scheduled trains during period $\tau$;

$y(\tau)$ : binary variables indicating the train pattern during period $\tau$, which equals to 1 if the large train pattern is adopted and 0 otherwise.

It is obvious that a train schedule for the intercity rail line can be transformed to calculate a set $\Omega=\{(x(\tau), y(\tau)) \mid \tau \in \mathfrak{R}\}$. In order to formulate a model accurately, two other hypotheses are presented as follows. At first, the travel passengers between two stations arrive uniformly at their origin station during a given period. Secondly, the passengers during a period are to be carried by the trains scheduled at the same period. The following notations 
and parameters are defined for constructing a train scheduling model for the intercity rail line:

$C_{1}$ : train capacity with large pattern (e.g., 600 persons);

$C_{0}$ : train capacity with small pattern (e.g., 340 persons);

$h_{\min }$ : prespecified minimum interval between two consecutive trains at the same station (e.g., $5 \mathrm{~min})$;

$h_{\text {max }}$ : prespecified maximum interval between two consecutive trains at the same station (e.g., $30 \mathrm{~min}$ );

$N_{1}$ : number of provided train-units with large pattern at the origin station;

$N_{0}$ : number of provided train-units with small pattern at the origin station;

$Q_{j}^{i}(\tau)$ : number of in-train passengers while train $j$ departs from station $i$ during period $\tau$;

$P_{j}^{i, i+s}(\tau)$ : number of passengers boarded on train $j$ who arrive at station $i$ travelling to station $i+s$ during period $\tau$.

\subsection{Constraints}

(1) Train Operation Constraint. The minimum interval between two consecutive trains should be required to ensure the operation safety of trains, while the predetermined maximum interval should not be broken for the passenger waiting times at the stations cannot be too long. Considering that the number of trains $x(\tau)$ is scheduled during period $\tau$, the same headway between two consecutive trains during this period is thus denoted by $60 / x(\tau)(\mathrm{min})$. As a result, the train operation constraint can be expressed by the following inequality:

$$
h_{\min } \leq \frac{60}{x(\tau)} \leq h_{\max }
$$

(2) Demand and Supply Constraint. According to the second hypothesis, the passenger demands generated during a period should be fulfilled by the trains scheduled at the same period. The demand and supply constraint is determined by the following:

$$
\sum_{j=1}^{x(\tau)} P_{j}^{i, i+s}(\tau)=D^{i, i+s}(\tau)
$$

(3) The In-Train Passengers. When train $j$ departs from station $i$ during period $\tau$, the number of in-train passengers contains two parts: one is the boarded passengers whose destinations are farther than station $i$, and the other is the passengers boarding at station $i$. Thus, the in-train passengers can be calculated as follows.

$$
Q_{j}^{i}(\tau)=\sum_{i_{1}=1 i_{2}=i+1}^{i} \sum_{j}^{K} P_{j}^{i_{1}, i_{2}}(\tau) .
$$


(4) The Fleet-Size Constraint. For the intercity rail transit lines with high-frequency train schedules, it is very important to have enough train units for dispatching at any moment. This paper considers that the fleet size is the major resource constraint in our scheduling design problem. Considering that the number of train units available at the origin station is known in advance, the fleet size constraint can be expressed as follows:

$$
\sum_{\tau \in \mathfrak{R}} x(\tau) \cdot[k \cdot y(\tau)+(1-k) \cdot(1-y(\tau))] \leq k \cdot N_{k}+(1-k) \cdot N_{1-k} \quad(k=1,0) .
$$

(5) The Number of Boarded Passengers. The number of boarded passengers traveling from station $i$ to station $i+s$ for train $j$ during period $\tau, P_{j}^{i, i+s}(\tau)$, is actually to assign the passengers to different trains. Considering that the passengers arrive uniformly at the original station and the trains associated with a given period are scheduled with a constant headway, the passenger demands should be evenly assigned to the trains during the period. The following formula is thus achieved for calculating the number of boarded passengers:

$$
P_{j}^{i, i+s}(\tau)= \begin{cases}{\left[\frac{D^{i, i+s}(\tau)}{x(\tau)}\right]} & \text { if } 1 \leq j \leq x(\tau)-R \\ {\left[\frac{D^{i, i+s}(\tau)}{x(\tau)}\right]+1} & \text { if } x(\tau)-R<j \leq x(\tau),\end{cases}
$$

where $[\cdot]$ means rounding, $D^{i, i+s}(\tau)=\left[D^{i, i+s}(\tau) / x(\tau)\right] \cdot x(\tau)+R, 0 \leq R \leq x(\tau)-1$.

\subsection{Objective Function}

The objective function is to minimize the total costs, which are composed of the waiting times of passengers at stations and the in-train crowded costs.

A constant interval between two consecutive trains is $60 / x(\tau)$ during period $\tau$ for there are $x(\tau)$ trains operating at this period. If the passengers arrive at station $i$ with uniform distribution, the average waiting time of each passenger during the period is $30 / x(\tau)$. The number of passengers arriving at station $i$ during the period is $\sum_{s=1}^{K-i} D^{i, i+s}(\tau)$, and the total waiting times of passengers at station $i$ is $(30 / x(\tau)) \cdot \sum_{s=1}^{K-i} D^{i, i+s}(\tau)$. Thus, the total waiting times of passengers during period $\tau$ can be expressed as follows:

$$
W(\tau)=\sum_{i=1}^{K-1} \sum_{s=1}^{K-i} \frac{30}{x(\tau)} \cdot D^{i . i+s}(\tau)
$$

This study introduces the in-train crowded cost to evaluate travel condition in the trains. The cost is incurred while the number of onboard passengers exceeds the maximum loading capacity of a train. As a result, the in-train crowded cost of train $j$ running between station $i$ and station $i+1$ during period $\tau$ is $Q_{j}^{i}(\tau) \cdot R^{i}$ if

$$
Q_{j}^{i}(\tau)> \begin{cases}C_{1} & \text { if } y(\tau)=1 \\ C_{0} & \text { if } y(\tau)=0\end{cases}
$$


and 0 otherwise. The total in-train crowded cost during period $\tau, F(\tau)$, is thus presented as follows:

$$
F(\tau)=\sum_{j=1}^{x(\tau)} \sum_{i=1}^{K-1} Q_{j}^{i}(\tau) \cdot R^{i} \cdot \delta\left[Q_{j}^{i}(\tau)-\left(y(\tau) \cdot C_{1}+(1-y(\tau)) \cdot C_{0}\right)\right]
$$

where $\delta(u)$ is the sign function which is equal to 1 if $u>0$ and 0 otherwise.

According to the above discussions, the objective function for minimizing the waiting times of passengers at stations and the in-train crowded costs can be expressed as follows:

$$
\min Z=\sum_{\tau \in \mathfrak{R}}[F(\tau)+W(\tau)]
$$

\section{Solution Algorithm}

The proposed model is a nonlinear programming problem which associates the tightly related zero-one and integer variables. It can hardly be solved with conventional gradient-based methods or commercial optimization solvers. Based on the mechanics of natural selection and natural genetics, genetic algorithm [16-18] is therefore adopted to solve the model developed in this study. The appeal of genetic algorithm comes from its simplicity and elegance as robust search algorithm as well as from its power to discover good solutions rapidly for difficult high-dimensional problems. In practicular, a hybrid procedure with two-layer framework is designed to solve the proposed model.

\subsection{Encoding Approach}

A special coding approach with two-layer structure, which includes two layers of decision variables, namely, the number of trains and train pattern, is adopted to solve the model. A day is divided equally into several short periods (e.g., one hour as a period in this study), and the length of a chromosome is represented by the number of periods. An integer embedded with the upper layer encoding indicates the number of trains scheduled during the given period. A binary number embedded with the lower layer encoding means the train pattern, where 1 indicates the large pattern and 0 indicates the small pattern.

In view of the headway constraint, the value of integers associated with the upper layer encoding, and the number of scheduled trains, should be located within $\left[60 / I_{\max }, 60 / I_{\min }\right]$. The number of scheduled trains $x(\tau)$ should range from 2 to 12 , for example, if $I_{\min }=5 \mathrm{~min}$ and $I_{\max }=30 \mathrm{~min}$. Figure 2 illustrates a chromosome using twolayer coding approach, where a day is divided into 10 periods, the number of scheduled trains is $6,11,7,6,9,5,4,2,8,5$, and the train pattern is large, large, small, small, large, large, small, small, large, small, respectively, during the concerned period. Following the abovementioned method, we can generate the initial chromosomes at random, then determine the number of trains $x(\tau)$ and the train pattern $y(\tau)$ and, finally, calculate the relevant parameters and the objective function. 


\begin{tabular}{|c|c|c|c|c|c|c|c|c|c|c|c|}
\hline Upper layer & 6 & 11 & 7 & 6 & 9 & 5 & 4 & 2 & 8 & 5 & The number of trains \\
\hline Lower layer & 1 & 1 & 0 & 0 & 1 & 1 & 0 & 0 & 1 & 0 & Train pattern \\
\hline
\end{tabular}

Figure 2: The illustration of a chromosome with two-layer coding approach.

\begin{tabular}{|c|c|c|c|c|c|c|c|c|c|c|c|c|c|c|c|c|c|c|c|c|c|}
\hline $\begin{array}{l}\text { The number of } \\
\text { trains }\end{array}$ & 5 & 11 & 8 & 6 & 9 & 5 & 7 & 2 & 5 & 7 & Adjust & 4 & 9 & 7 & 5 & 8 & 4 & 6 & 2 & 4 & 6 \\
\hline Train pattern & 1 & 0 & 0 & 1 & 1 & 1 & 0 & 0 & 1 & 0 & & 1 & 0 & 0 & 1 & 1 & 1 & 0 & 0 & 1 & 0 \\
\hline
\end{tabular}

Figure 3: The adjustment of an infeasible chromosome.

\subsection{Feasibility Adjustment}

It is necessary to pay special attention to the fleet size constraint in the proposed model, because the number of trains departing from the original station during one day should not exceed the provided train units, and the chromosomes are required to adjust the feasibility as the execution process of the procedure. The total number of trains with large pattern is calculated by checking the train pattern whose encoding is 1 at the lower layer. The number of trains $x(\tau)$ associated with period $\tau$ should be reduced by 1 with descending order if the total number of trains exceeds the available train units $N_{1}$, until the condition is satisfied. The total number of trains with small pattern is required to check its feasibility similarly. All other chromosomes generated at the iterative process of genetic operation should be also adjusted with the above-mentioned method. For example, a new chromosome as shown in Figure 3 can be obtained after the adjustment of the original one if the number of available train units $N_{1}$ and $N_{0}$ are 25 and 30, respectively.

\subsection{Fitness Function}

By calculating the value of objective function, we can get the fitness of each chromosome. The formulation can be expressed as follows:

$$
\text { fitness }=\frac{Z_{\max }-Z}{Z_{\max }-Z_{\min }}
$$

where $Z$ is the objective from (2.9), $Z_{\max }$ and $Z_{\min }$ denote, respectively, the maximum and minimum values of the objectives associated with the current generation.

\subsection{Crossover Operator}

Considering that two-layer encoding approach is adopted in this paper, a layered crossover operation with double probabilities is proposed. The procedure of the layered crossover operation is presented as follows.

Algorithm 3.1. The layered crossover operation consists of the following steps. 


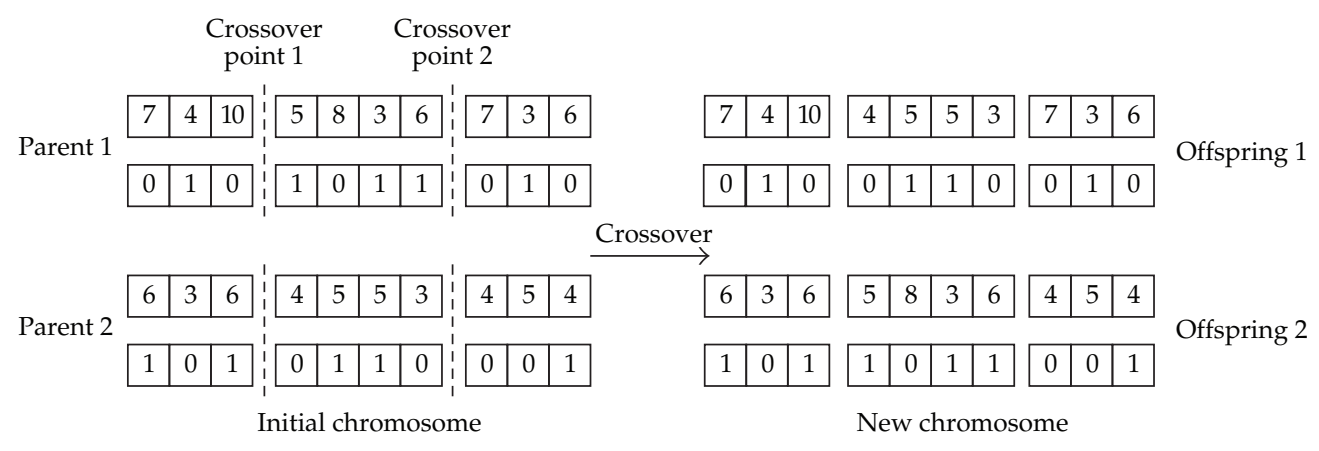

Figure 4: The illustration of two-layer crossover operation.

Step 1. Two crossover probabilities $P_{c}^{1}$ and $P_{c}^{2}$ are set to decide the upper layer operation and the lower layer operation, and a random number $\theta$ with uniform distribution at $[0,1]$ is generated to indicate the judgment criterion.

Step 2. Two crossover points are selected randomly, then two gene strings between two crossover points on the parent chromosomes are exchanged with each other.

Step 3. Two new offspring chromosomes are generated by exchanging the corresponding gene strings of the parent chromosomes if $\theta \leq \min \left\{P_{c}^{1}, P_{c}^{2}\right\}$, which means that two gene strings associated with the upper layer and the lower layer are exchanged simultaneously between parent 1 and parent 2 . If $P_{c}^{2}<\theta \leq P_{c}^{1}$, only the gene strings associated with the upper layer of two parents take the crossover operation, and the gene strings associated with the lower layer remain the same. If $P_{c}^{1}<\theta \leq P_{c}^{2}$, only the gene strings associated with the lower layer of two parents take the crossover operation, and the gene strings associated with the upper layer remain the same.

Assume that the crossover probability $\theta \leq \min \left\{P_{c}^{1}, P_{c}^{2}\right\}$, the layered crossover operation can be demonstrated by Figure 4 .

\subsection{Mutation Operator}

According to the characteristics of the problem, the large train pattern is suitable for the period with larger number of trains, while the small train pattern is suitable for the period with less number of trains. A layered mutation operation with a single point is then proposed in the paper. Firstly, a gene associated with the upper layer encoding takes mutation operation, and the corresponding gene with the lower layer is then operated by the result of the upper layer. The procedure of the layered mutation operation is summarized as follows.

Algorithm 3.2. The layered mutation operation consists of the following steps.

Step 1. Two mutation probabilities $P_{m}^{1}$ and $P_{m}^{2}$ are set to decide the mutation operations associated with the upper layer and the lower layer, respectively, denoting the judgment criteria of the mutation operation with upper and lower layers, and a random number $\theta$ with uniform distribution at $[0,1]$ is generated to indicate the judgment criterion. 


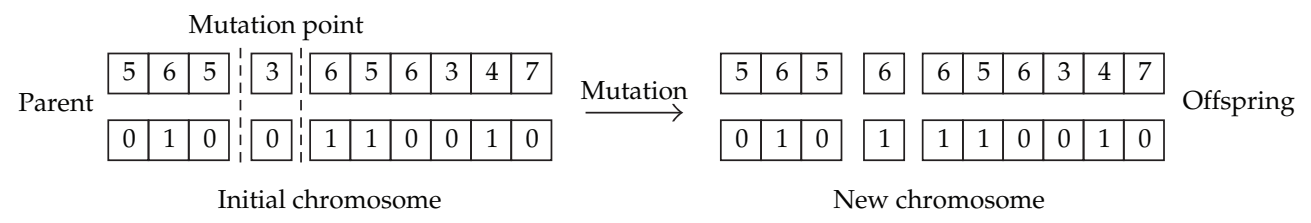

Figure 5: The illustration of the layered mutation operation.

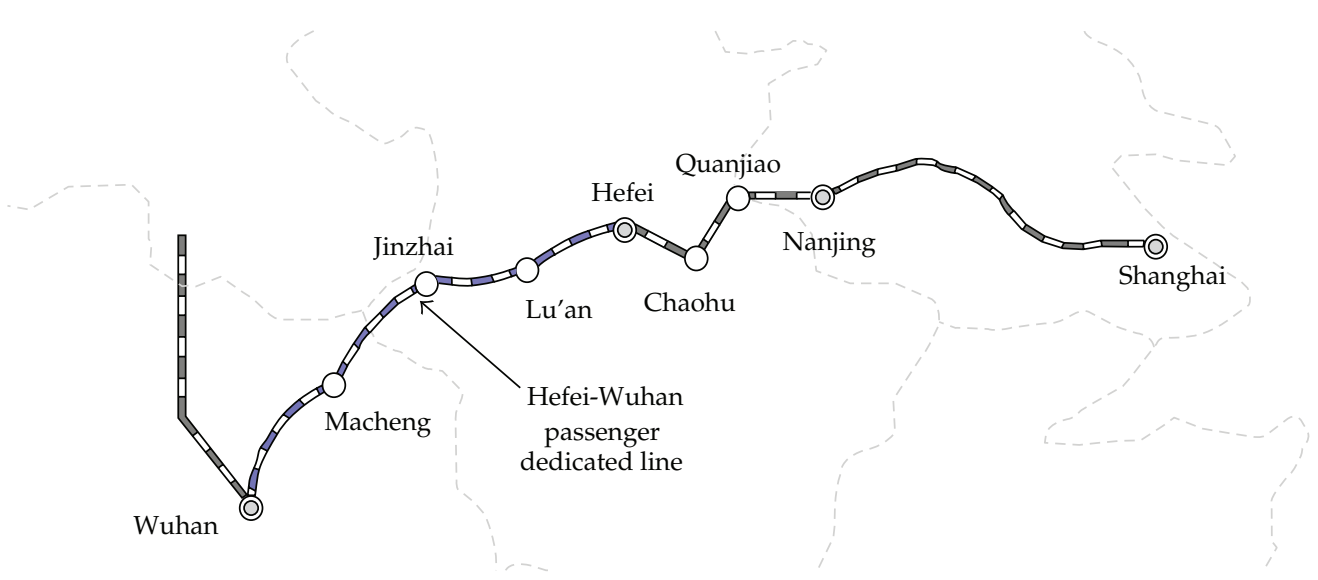

Figure 6: The illustration of Hefei-Wuhan intercity rail line.

Step 2. A mutation point is selected randomly, and then the genes associated with the upper layer and lower layer are mutated.

Step 3. The selected gene at the upper layer of the parent chromosome is replaced with another number which is located within the predetermined range if $\theta \leq P_{m}^{1}$. The mutation operation with the lower layer will be determined by the result of the upper layer if $\theta \leq P_{m}^{2}$. If the value of the gene at the upper layer encoding increases, the value of the corresponding gene at the lower layer should be changed from 0 to 1 . If the value of the gene at the upper layer decreases, the value of the corresponding gene at the lower layer encoding should replace 1 with 0 .

Assume, for example, that the mutation probability is $\theta \leq P_{m}^{1}$ and $\theta \leq P_{m}^{2}$, the layered mutation operation can be demonstrated by Figure 5 .

\section{Numerical Example}

\subsection{Line}

Hefei-Wuhan intercity rail line, which has operated since April, 2009, is an important passenger dedicated railway line between Hefei city and Wuhan city in China. The line has a total length of 364 kilometers with the designed speed of $250 \mathrm{~km} / \mathrm{h}$. There are 5 stations along the line, namely Wuhan station, Macheng North station, Jinzhai station, LiuAn station, and Hefei station as shown in Figure 6. 


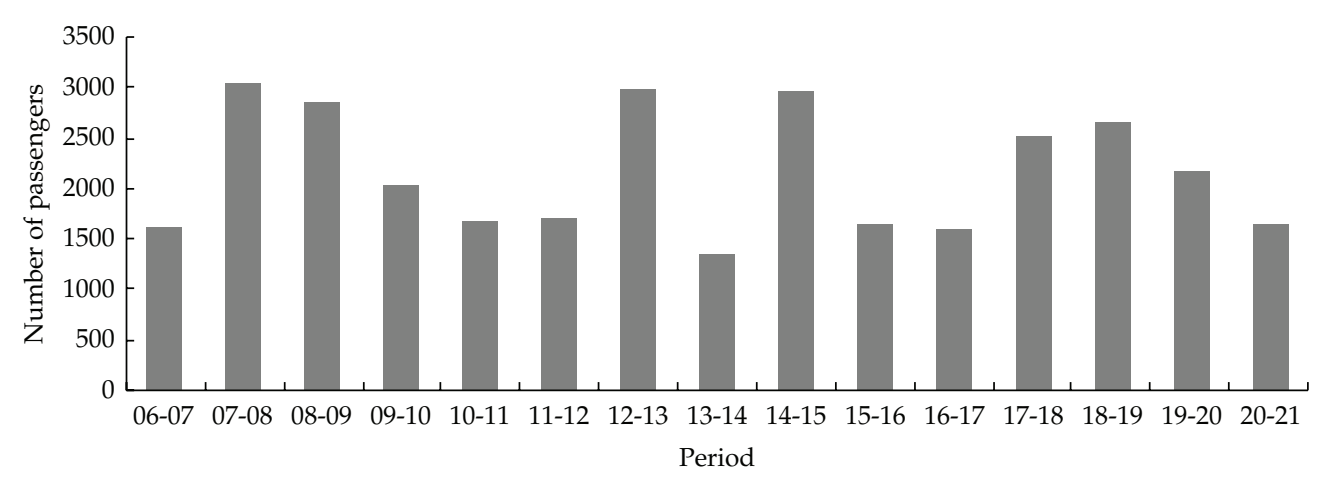

Figure 7: The period-dependent passenger demands counted in terms of one hour.

Table 1: The running time between two adjacent stations (min).

\begin{tabular}{lccc}
\hline Adjacent stations & Running time & Adjacent stations & Running time \\
\hline Wuhan-Macheng North & 52 & Jinzhai-Liuan & 22 \\
Macheng North-Jinzhai & 44 & Liuan-Hefei & 32 \\
\hline
\end{tabular}

\subsection{Demands}

With a particular focus on a typical weekday, the example below considers the operation from 6:00 AM to 21:00 PM at the origin station. The period-dependent passenger demands, as shown in Figure 7, are illustrated by the total number of passengers arriving at the stations during each one hour.

\subsection{Results}

The running times between two adjacent stations for Hefei-Wuhan intercity rail line are given in Table 1. The capacities associated with two train patterns $C_{1}$ and $C_{0}$ are 600 and 340, respectively, and the fleet sizes of available train units $N_{1}$ and $N_{0}$ at the origin station are 40 and 50, respectively. The prespecified minimum and maximum headways $I_{\min }$ and $I_{\max }$ are 5 and 30 minutes, respectively.

The parameter values for the layered genetic algorithm are listed as follows. The population size is 100 and the number of total iterations is 300 . The crossover probabilities $P_{c}^{1}$ and $P_{c}^{2}$ are 0.90 and 0.95 , and the mutation probabilities $P_{m}^{1}$ and $P_{m}^{2}$ are 0.10 and 0.30 , respectively. After 252 iterations, a train schedule for Hefei-Wuhan intercity rail line from 6:00 AM to 21:00 PM is obtained by the developed procedure, which is shown in Table 2, and the trend of objective value in processing the algorithm can be shown in Figure 8.

From Table 2, we can see that the total number of trains scheduled during the operation period is 77, of which the number of trains with large pattern is 37 , and the number of trains with small pattern is 40 . The average full-load rate of trains is $95.65 \%$, which means the optimized train schedule could both economize the operation cost for the railway department and provide comfortable travel environment for the passengers. The number of scheduled trains and the train pattern during each period are shown in Figure 9.

We can see from Figure 9 that the number of scheduled trains is generally proportional to passenger demand, which means that the number of trains during high-peak periods is 


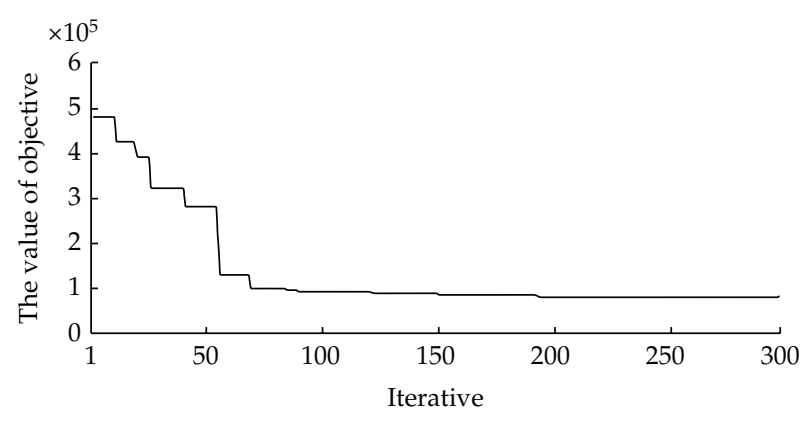

Figure 8: The variation curve on the objective with iterations.

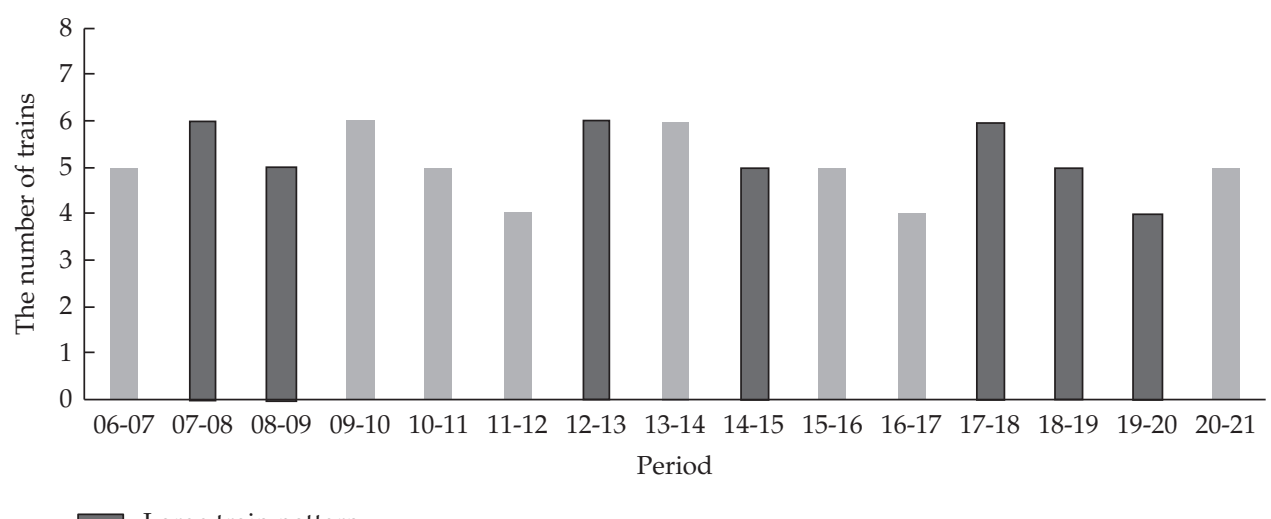

Large train pattern

Small train pattern

Figure 9: The number of scheduled trains and the train-pattern during each period.

Table 2: The number of trains and train pattern during each period.

\begin{tabular}{lccc}
\hline Period & Number of trains & Train pattern & Average load-rate (\%) \\
\hline 6:00-7:00 & 5 & 0 & 96.00 \\
7:00-8:00 & 6 & 1 & 85.14 \\
8:00-9:00 & 5 & 1 & 95.50 \\
9:00-10:00 & 6 & 0 & 99.71 \\
10:00-11:00 & 5 & 0 & 99.24 \\
11:00-12:00 & 4 & 0 & 99.88 \\
12:00-13:00 & 6 & 1 & 83.64 \\
13:00-14:00 & 6 & 0 & 90.69 \\
14:00-15:00 & 5 & 1 & 99.57 \\
15:00-16:00 & 5 & 0 & 97.53 \\
16:00-17:00 & 4 & 0 & 94.71 \\
17:00-18:00 & 6 & 1 & 70.58 \\
18:00-19:00 & 5 & 1 & 89.57 \\
19:00-20:00 & 4 & 1 & 90.21 \\
20:00-21:00 & 5 & 0 & 97.53 \\
\hline
\end{tabular}


larger and vice versa. The train pattern is mainly with large pattern during the high-peak periods, and during the low-peak periods the train pattern is mainly with small pattern.

\section{Conclusion}

This paper proposes a phase-regular scheduling method for an intercity rail line, which divides an operational day evenly into several time blocks and applies a regular traindeparting interval and the same train length for each period. A nonlinear mixed zero-one programming model, which could accurately calculate the passenger waiting time and the in-train crowded cost, is established. A hybrid genetic algorithm with two-layer framework is designed to solve the proposed model. Finally, the validation of the model and the algorithm has been tested with the application of Hefei-Wuhan intercity rail line in China. The results show that the proposed method can effectively solve the scheduling problem of intercity rail lines. Considering the modeling details which are closer to reality, such as under a random or fuzzy environment, is an important topic for further research. At the same time, there is the necessity to explore the response of passengers to the optimized schedule and to extend the method to a network case.

\section{Acknowledgments}

The work described in the paper was supported by National Natural Science Foundation of China (no. 50968009, no. 71261014, and no. 51210305046) and the Research Fund for the Doctoral Program of Higher Education (no. 20096204110003).

\section{References}

[1] N. S. A. Ghoneim and S. C. Wirasinghe, "Optimum zone structure during peak periods for existing urban rail lines," Transportation Research B, vol. 20, no. 1, pp. 7-18, 1986.

[2] J. W. Goossens, S. van Hoesel, and L. Kroon, "On solving multi-type railway line planning problems," European Journal of Operational Research, vol. 168, no. 2, pp. 403-424, 2006.

[3] C. Liebchen, "The first optimized railway timetable in practice," Transportation Science, vol. 42, no. 4, pp. 420-435, 2008.

[4] M. T. Claessens, N. M. Van Dijk, and P. J. Zwaneveld, "Cost optimal allocation of rail passenger lines," European Journal of Operational Research, vol. 110, no. 3, pp. 474-489, 1998.

[5] K. Ghoseiri, F. Szidarovszky, and M. J. Asgharpour, "A multi-objective train scheduling model and solution," Transportation Research B, vol. 38, no. 10, pp. 927-952, 2004.

[6] M. B. Khan and X. Zhou, "Stochastic optimization model and solution algorithm for robust doubletrack train-timetabling problem," IEEE Transactions on Intelligent Transportation Systems, vol. 11, no. 1, pp. 81-89, 2010.

[7] X. Zhou and M. Zhong, "Single-track train timetabling with guaranteed optimality: branch-andbound algorithms with enhanced lower bounds," Transportation Research B, vol. 41, no. 3, pp. 320-341, 2007.

[8] K. Nachtigall and S. Voget, "Minimizing waiting times in integrated fixed interval timetables by upgrading railway tracks," European Journal of Operational Research, vol. 103, no. 3, pp. 610-627, 1997.

[9] A. de Palma and R. Lindsey, "Optimal timetables for public transportation," Transportation Research B, vol. 35, no. 8, pp. 789-813, 2001.

[10] S. Nguyen, S. Pallottino, and F. Malucelli, "A modeling framework for passenger assignment on a transport network with timetables," Transportation Science, vol. 35, no. 3, pp. 238-249, 2001.

[11] R. C. W. Wong, T. W. Y. Yuen, K. W. Fung, and J. M. Y. Leung, “Optimizing timetable synchronization for rail mass transit," Transportation Science, vol. 42, no. 1, pp. 57-69, 2008. 
[12] L. Meng and X. Zhou, "Robust single-track train dispatching model under a dynamic and stochastic environment: a scenario-based rolling horizon solution approach," Transportation Research B, vol. 45, no. 7, pp. 1080-1102, 2011.

[13] M. Carey and I. Crawford, "Scheduling trains on a network of busy complex stations," Transportation Research B, vol. 41, no. 2, pp. 159-178, 2007.

[14] G. Caimi, F. Chudak, M. Fuchsberger, M. Laumanns, and R. Zenklusen, "A new resource-constrained multicommodity flow model for conflict-free train routing and scheduling," Transportation Science, vol. 45, no. 2, pp. 212-227, 2011.

[15] Y. H. Chang, C. H. Yeh, and C. C. Shen, "A multiobjective model for passenger train services planning: application to Taiwan's high-speed rail line," Transportation Research B, vol. 34, no. 2, pp. 91-106, 2000.

[16] M. Gen and R. W. Cheng, Genetic Algorithms and Engineering Optimization, John Wiley \& Son, New York, NY, USA, 2000.

[17] H. M. Niu, "Determination of the skip-stop scheduling for a congested transit line by bilevel genetic algorithm," International Journal of Computational Intelligence Systems, vol. 4, no. 6, pp. 1158-1167, 2011.

[18] J. Gao,, R. Chen, and Q. Pan, "A hybrid genetic algorithm for the distributed permutation flowshop scheduling problem," International Journal of Computational Intelligence Systems, vol. 4, no. 4, pp. 497508, 2011. 


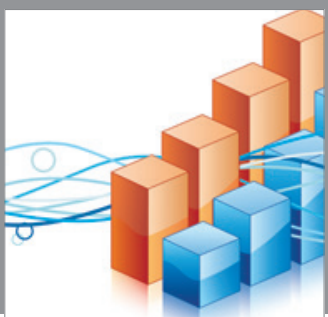

Advances in

Operations Research

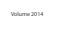

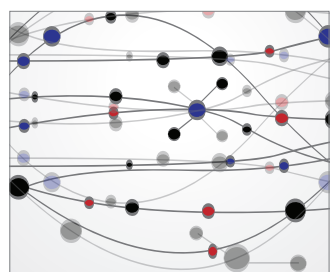

\section{The Scientific} World Journal
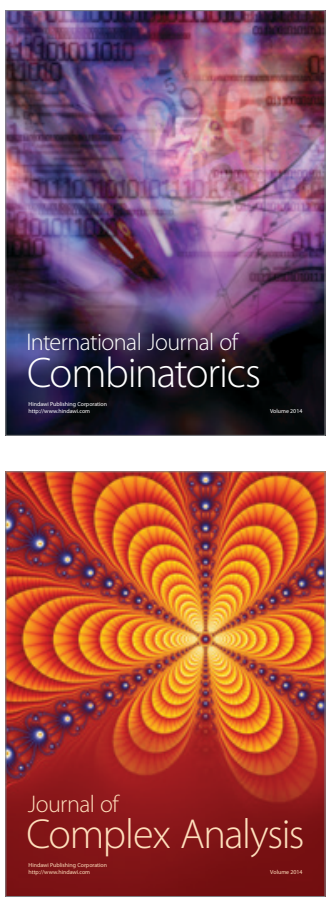

International Journal of

Mathematics and

Mathematical

Sciences
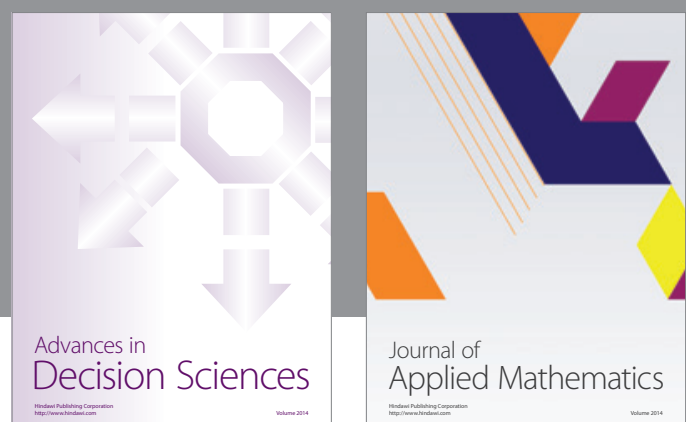

Journal of

Applied Mathematics
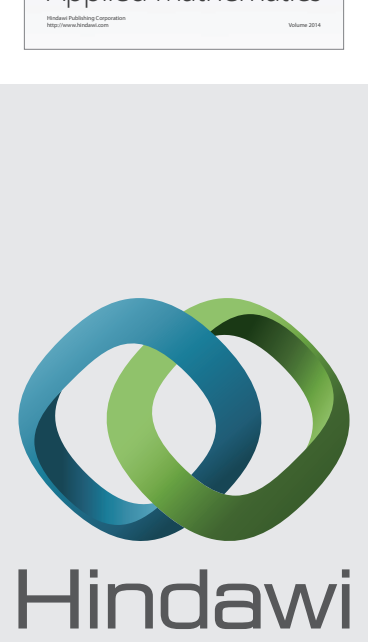

Submit your manuscripts at http://www.hindawi.com
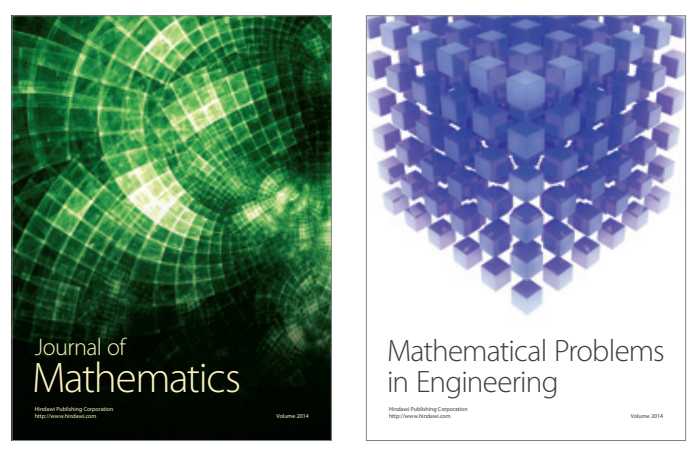

Mathematical Problems in Engineering
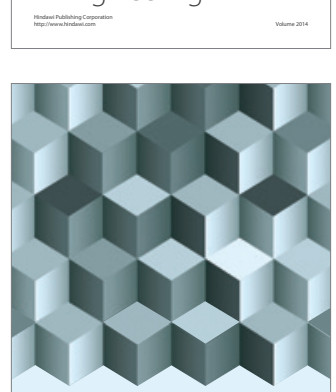

Journal of

Function Spaces
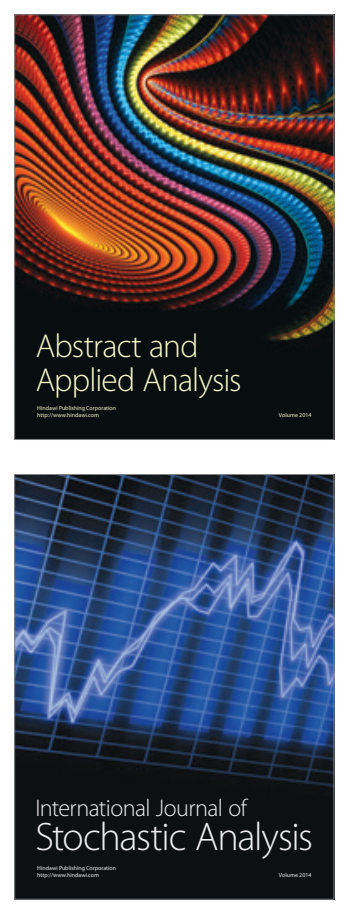

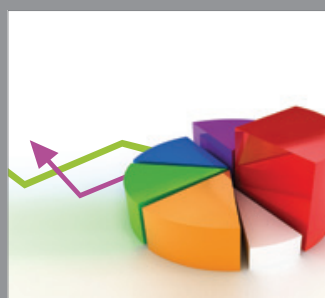

ournal of

Probability and Statistics

Promensencen
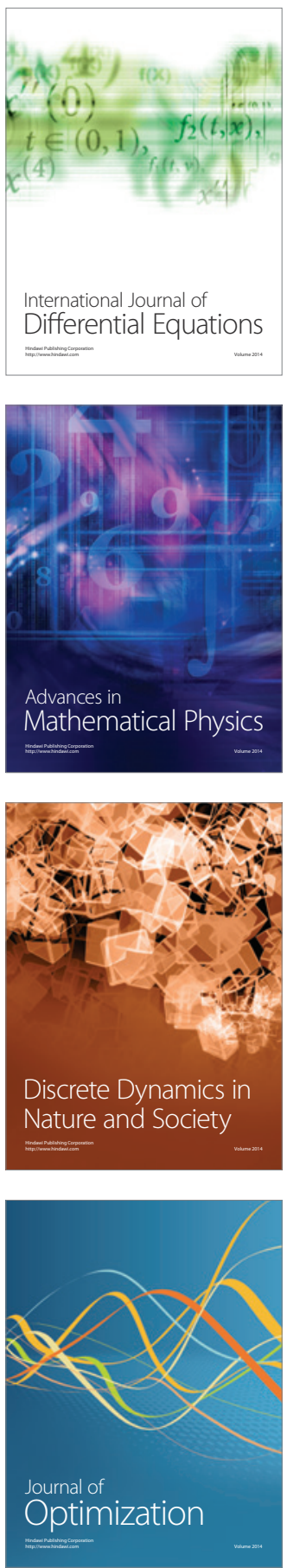